\title{
Octreotide long-acting repeatable and lanreotide Autogel are equally effective in controlling growth hormone secretion in acromegalic patients
}

\author{
S W van Thiel, J A Romijn, N R Biermasz, B E P M Ballieux ${ }^{1}$, M Frölich ${ }^{1}$, J W A Smit, E P M Corssmit, F Roelfsema \\ and A M Pereira \\ Department of Endocrinology and Metabolism and ${ }^{1}$ Department of Clinical Chemistry, Leiden University Medical Centre, Leiden, The Netherlands \\ (Correspondence should be addressed to $S W$ van Thiel, Department of Endocrinology, C4-R, Leiden University Medical Centre, PO Box 9600,2300 RC \\ Leiden, The Netherlands; Email: s.w.van_thiel@lumc.nl)
}

\begin{abstract}
Objective: Recently a new depot preparation of the long-acting somatostatin analogue, lanreotide Autogel was introduced for the treatment of acromegaly. Like octreotide long-acting repeatable (LAR), it has high binding affinity for the somatostatin receptor subtype SSTR 2 and less binding affinity for SSTR 5 . We hypothesized that the ability to suppress growth hormone (GH) secretion in patients with acromegaly would be similar for these depot preparations.

Patients and study design: Seven patients (mean age \pm s.E.M. $48.4 \pm 7$ years) on long-term octreotide LAR treatment at a monthly injection interval for a mean of 2.8 years were enrolled in the study. They underwent a GH secretory profile study with $10 \mathrm{~min}$ sampling for $24 \mathrm{~h}, 28$ days after an injection. At 2, 4 and 6 weeks after the next injection fasting GH profiles (every $30 \mathrm{~min}$ for $3.5 \mathrm{~h}$ ) and serum IGF-I measurements were measured. These investigations were repeated 12 months later, when the patients were on an individually titrated stable dose of lanreotide Autogel.

Results: Secretory characteristics and total $24 \mathrm{~h} \mathrm{GH}$ secretion, estimated by deconvolution analysis of the $10 \mathrm{~min} 24 \mathrm{~h}$ plasma GH concentrations, did not show differences between these two long-acting somatostatin analogues. Both drugs were equally effective in GH and IGF-I suppression as measured at 2, 4 and also at 6 weeks following an injection.

Conclusion: The efficacy of lanreotide Autogel and octreotide LAR was equal, notwithstanding that these drugs are administered in a different way and have different pharmacokinetics.
\end{abstract}

European Journal of Endocrinology 150 489-495

\section{Introduction}

Acromegaly is a syndrome caused by overproduction of growth hormone $(\mathrm{GH})$ from a $\mathrm{GH}$-secreting pituitary adenoma. The high levels of $\mathrm{GH}$ and insulin-like growth factor I (IGF-I) are associated with increased morbidity and mortality, which necessitates adequate control of the disease $(1,2)$. Although transsphenoidal microsurgery is the first-choice treatment in eligible patients, somatostatin analogues are the preferred secondary therapy in the $30-40 \%$ of patients not in remission after surgery and in the $10-15 \%$ who develop recurrence of disease during long-term followup $(1,3,4)$. Furthermore, primary treatment of acromegaly with somatostatin analogues is increasingly applied (5).

Somatotroph adenomas express high levels of somatostatin receptor subtypes SSTR 2 and $5(6,7)$. The somatostatin analogues octreotide (Novartis Pharma AG, Basel, Switzerland) and lanreotide (Ipsen
Biotech, Paris, France) both have high binding affinity for the SSTR 2 and to a lesser extent for the SSTR 5 (8). Octreotide long-acting repeatable (LAR), the depot preparation of octreotide given by monthly i.m. injections, is able to normalize $\mathrm{GH}$ concentrations in $56 \%$ and IGF-I in $66 \%$ of the patients (9). Lanreotide slow release (SR), the more frequently i.m. injected depot preparation of lanreotide, seems less effective in normalizing GH and IGF-I concentration (10-13). Recently, lanreotide Autogel, a new slow-release depot preparation of lanreotide was introduced. This new delivery formulation is available in small-volume, prefilled syringes, and is administrated by monthly deep s.c. injections (14).

Considering the comparable binding affinity of octreotide and lanreotide for SSTRs, we hypothesized that there would be no difference between the two depot preparations in their ability to suppress GH secretion in patients with acromegaly. To test this hypothesis, we applied two different independent 
approaches: first by measuring GH secretion characteristics via deconvolution analysis of $24 \mathrm{~h}$ plasma $\mathrm{GH}$ concentrations profiles. We have recently shown that sustained blockade by octreotide can not restore all these parameters of abnormal GH secretion, but we have not established that for lanreotide (15). Secondly, we evaluated the extent and duration to which both depot somatostatin analogues suppressed GH and IGF-I secretion.

\section{Subjects and methods}

\section{Patients}

For this study we included seven patients with active acromegaly (of whom two were studied previously), who showed relatively good responsiveness to octreotide (15). The diagnosis was based on the characteristic clinical features and confirmed by insufficient suppression of GH concentration during the oral glucose tolerance test (OGTT), the presence of a pituitary adenoma on radiological imaging, and elevated age-adjusted IGF-I concentrations. The clinical characteristics of the patients are described in Table 1. All patients used octreotide LAR (20 or $30 \mathrm{mg}$, at a monthly scheme) for an average duration of 2.8 years. None of the patients received a dopamine agonist before or during the study.

Although the treatment goals were similar for both analogues, i.e. $\mathrm{GH}<5 \mathrm{mU} / \mathrm{l}$ and a normal age-related IGF-I, four out of seven patients were using $20 \mathrm{mg}$ octreotide LAR, whereas six patients required the highest dose $(120 \mathrm{mg})$ of lanreotide. The patients were first titrated on octreotide LAR. When there was a discrepancy between GH and IGF-I concentrations we gave preference to IGF-I, at least when the clinical response was satisfactory. The resultant octreotide LAR dose required was $20 \mathrm{mg}$ in four, and $30 \mathrm{mg}$ in three patients. Three out of four patients already had reached the treatment goals, and the fourth patient did not have better results on $30 \mathrm{mg}$ octreotide LAR.

The local Medical Ethical Committee approved the protocol and all patients gave written informed consent.

\section{Study protocol (Fig. 1)}

The patients were investigated in a prospective study design according to the following protocol. The patients were first analysed on their regular octreotide LAR treatment. To assess details of GH secretory characteristics during chronic octreotide treatment, a $24 \mathrm{~h} \mathrm{GH}$ profile (with $10 \mathrm{~min}$ intervals) was performed 4 weeks after an octreotide LAR injection. To assess the extent and duration of GH suppression during chronic treatment, fasting morning $\mathrm{GH}$ profiles were obtained at 2, 4 and 6 weeks after the last octreotide LAR injection. Subsequently, patients were included in a Phase II International Multicentre Trial for Evaluating the Efficacy and Safety of Lanreotide Autogel (data on file, Ipsen-Beaufour, study E2852030717). Patients were randomized to receive 60 , 90 or $120 \mathrm{mg}$ lanreotide Autogel, the dose of which was subsequently adjusted according to individual fasting $\mathrm{GH}$ profiles and IGF-I concentrations during the course of the trial aiming at a serum GH concentration $<5 \mathrm{mU} / \mathrm{l}$ and a normal IGF-I for age. Six of the seven patients required the highest lanreotide dose. At the end of the international trial, 1 year later, the investigations were repeated, i.e. a 24 h plasma GH profile 4 weeks after a lanreotide Autogel injection followed by a GH profile 2, 4 and 6 weeks after an injection. Radiological imaging (magnetic resonance imaging or computed tomography scan) of the pituitary was performed before the start on each analogue and twice during follow-up.

\section{Study parameters}

Twenty-four hour GH profile Patients were hospitalized the evening before the sampling studies, 27 days after the last injection with octreotide LAR or lanreotide Autogel. The following morning, an i.v. cannula was inserted into a large forearm vein, and blood samples were withdrawn at $10 \mathrm{~min}$ intervals for the next $24 \mathrm{~h}$, starting at $0900 \mathrm{~h}$. Standard meals were served at predetermined time points, 0730, 1130 and $1730 \mathrm{~h}$. Lights were turned off between 2200 and $0700 \mathrm{~h}$. All plasma samples were frozen immediately and stored at $-20^{\circ} \mathrm{C}$ until analysis.

Table 1 Characteristics of seven patients studied during chronic octreotide LAR and lanreotide Autogel therapy.

\begin{tabular}{|c|c|c|c|c|c|c|c|c|c|}
\hline No. & Sex & $\begin{array}{c}\text { Age } \\
\text { (years) }\end{array}$ & $\begin{array}{c}\text { Transsphenoidal } \\
\text { surgery }\end{array}$ & Radiotherapy & $\begin{array}{l}\text { Years on } \\
\text { octreotide } \\
\text { LAR }\end{array}$ & $\begin{array}{c}\text { IGF-I at } \\
\text { presentation } \\
(\mathrm{nmol} / \mathrm{l})\end{array}$ & $\begin{array}{c}\text { Suppressed } \\
\text { GH during OGTT } \\
(\mathrm{mU} / \mathrm{l})\end{array}$ & $\begin{array}{l}\text { Octreotide } \\
\text { LAR dose } \\
\quad(\mathrm{mg})\end{array}$ & $\begin{array}{c}\text { Lanreotide } \\
\text { Autogel } \\
\text { dose (mg) }\end{array}$ \\
\hline 1 & $\mathrm{~F}$ & 57 & 1986 & No & 3.4 & 97 & 30 & 20 & 120 \\
\hline 2 & M & 32 & 1997 & No & 3.1 & - & 7.15 & 30 & 120 \\
\hline 3 & $M$ & 42 & 1995 & 1996 & 2.1 & 97 & 10.47 & 20 & 60 \\
\hline 4 & M & 53 & No & No & 3.5 & 37 & 196 & 20 & 120 \\
\hline 5 & M & 20 & No & No & 3.5 & 30 & - & 30 & 120 \\
\hline 6 & $M$ & 68 & No & No & 3.2 & 69 & 19.8 & 30 & 120 \\
\hline 7 & $M$ & 67 & No & No & 1.0 & 27 & 14.71 & 20 & 120 \\
\hline
\end{tabular}

—, data not available (test performed elsewhere). 


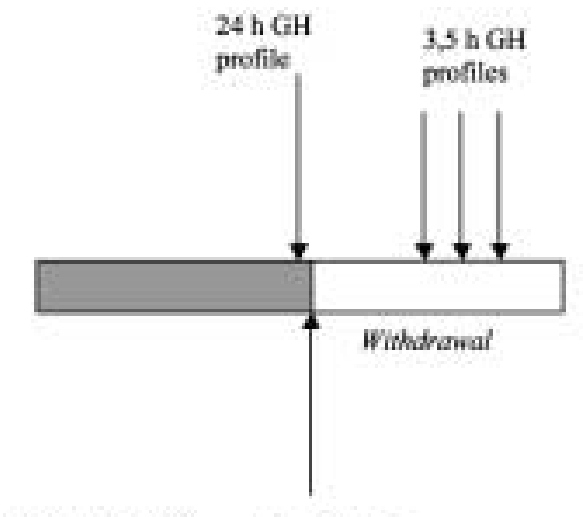

Oetrotide LAR Last injoction

Octreotide LAR

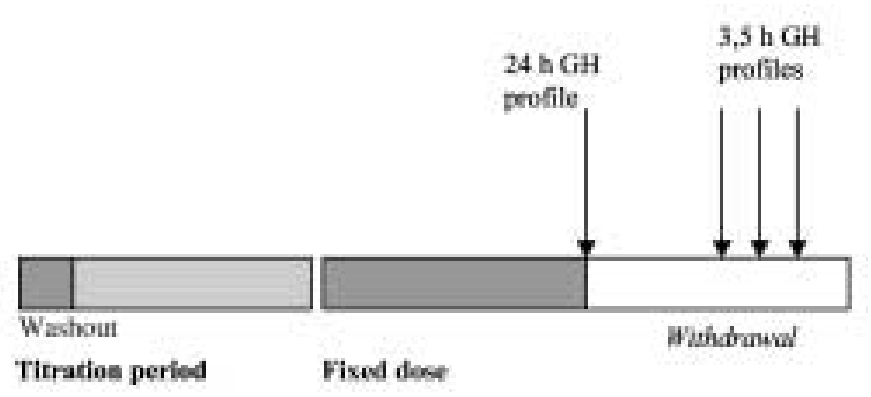

Last injection

Lanreotide Autogel

\begin{tabular}{l|l|l|l|l}
\hline $1-3.5 \mathrm{yss}$ & $6 \mathrm{wks}$ & ${ }_{10 \mathrm{wks}}$ & $1 \mathrm{yr}$ & $6 \mathrm{wks}$
\end{tabular}

Figure 1 Study protocol.

Short-withdrawal study After an overnight fast, patients were admitted to the Clinical Research Centre of the Department of Endocrinology between 0800 and $0900 \mathrm{~h}$. An i.v. catheter was inserted in a forearm vein for collection of all blood samples. The patients were fasting during the blood-sampling procedure. From the first blood sample, IGF-I concentration was determined. Subsequently, blood samples were obtained every $30 \mathrm{~min}$ for $3.5 \mathrm{~h}$. The mean $\mathrm{GH}$ was calculated from eight samples. All samples from the patients were stored at $-20{ }^{\circ} \mathrm{C}$ until analysis in the same GH and IGF-I assay runs.

\section{Assays}

GH concentrations were measured with a sensitive time-resolved fluoro-immunoassay (Wallac, Turku, Finland) specific for the $22 \mathrm{kDa} \mathrm{GH}$. The standard was recombinant human GH (Genotropin; KabiVitrium, Uppsala, Sweden), which was calibrated against the WHO First International Reference Preparation 80/505. (To convert $\mathrm{mU} / \mathrm{l}$ to $\mu \mathrm{g} / \mathrm{l}$, divide by 2.6 .) The limit of detection (defined as the value 2 S.D. above the mean value of the zero standard) was $0.03 \mathrm{mU} / \mathrm{l}$. The intra-assay coefficient of variation (CV) ranged from 1.6 to $8.4 \%$ in the assay range between 0.26 and $47 \mathrm{mU} / \mathrm{l}$, with corresponding inter-assay CV values of $2.0-9.9 \%$. The total serum IGF-I concentration was measured by IRMA after dissociation and blocking of the IGF-binding proteins with IGF-II (Nichols Institute Diagnostics, San Juan Capistrano, CA, USA). The reference values $(95 \%$ confidence intervals) per decade ranged from 11 to $77 \mathrm{nmol} / \mathrm{l}$ for 20-30 years, 10 to $42 \mathrm{nmol} / \mathrm{l}$ for $30-40$ years, 8 to
$33 \mathrm{nmol} / \mathrm{l}$ for $40-50$ years, 8 to $24 \mathrm{nmol} / \mathrm{l}$ for $50-60$ years and 8 to $22 \mathrm{nmol} / \mathrm{l}$ for $60-70$ years.

\section{Analytical techniques}

Multiparameter deconvolution analysis was used to quantify basal GH secretion and the GH half-life (16). This waveform-specific technique estimates the rate of basal release, the number and mass of randomly ordered secretory bursts, and the subject-specific (mono-exponential) half-life (17). The daily secretion rate is the product of secretory burst frequency and mean mass of GH released per event. Total GH secretion is the sum of basal and pulsatile secretion $(16,17)$.

\section{Statistical analysis}

Data are given as means \pm S.E.M., unless otherwise noted. Statistical analysis was carried out using Student's $t$-test when applicable and with multivariate repeat-measures analysis to compare differences between and within groups. Calculations were performed with SPSS for Windows version 11.0 (SPSS, Inc., Chicago, IL, USA). $P<0.05$ was considered significant.

\section{Results}

All patients completed the study. Chronic treatment with lanreotide Autogel was well tolerated by all patients, as was octreotide LAR treatment. Three patients experienced bowel cramps and diarrhoea for several days after the first injections of lanreotide Autogel, resolving after the fourth injection. No adverse events were reported during the study. 
Of the three patients who had previous surgery, no change in residual tumour volume was noted on both analogues. In one out of four non-operated patients, a $50 \%$ decrease in tumour volume was found during octreotide LAR treatment, but no further change in tumour volume could be detected in these patients during subsequent lanreotide treatment.

\section{Twenty-four hour plasma GH profile}

Figure 2 describes the $24 \mathrm{~h}$ plasma GH concentrations of all seven patients during treatment with both somatostatin analogues, showing a remarkably similar pattern. The secretory characteristics as analysed by deconvolution analysis were similar during both treatments and are detailed in Table 2. The pulsatile and total GH secretion per $24 \mathrm{~h}$ were not different between octreotide LAR and lanreotide Autogel.

\section{Short withdrawal study}

Table 3 describes the mean GH levels and IGF-I concentrations during the 6 weeks of withdrawal. Mean GH and IGF-I levels obtained at 2, 4 and 6 weeks were not different between the two treatment groups. Over time, no significant changes in GH levels and IGF-I concentrations could be detected within the two treatments.

The number of patients achieving control of both GH and IGF-I were three under both treatment modalities. In only one patient of the remaining four was $\mathrm{GH}$ normal on octreotide and slightly elevated on lanreotide, achieving concordance on both criteria in six out of seven patients.

Six weeks after a lanreotide Autogel and octreotide LAR injection only one patient had a normal mean GH and IGF-I for age.

\section{Discussion}

This study is the first report that compares lanreotide Autogel, a new slow-release formulation, with octreotide LAR. Both drugs are long-acting octapeptide depot somatostatin analogues used for the treatment of acromegaly $(14,18)$. Both analogues showed similarly suppressed $\mathrm{GH}$ levels during a detailed $24 \mathrm{~h}$ study. Moreover, during the regular injection interval ( 2 and 4 weeks) and also 6 weeks after the injection, both drugs equally suppressed mean serum GH and IGF-I concentrations.

The results from this study on lanreotide Autogel differ from previous studies, comparing lanreotide SR with octreotide LAR. Octreotide LAR, using 1-monthly injections generally seems to show a higher efficacy in suppressing serum GH and IGF-I levels than lanreotide SR, injected i.m. every 7-21 days (10-13). Lanreotide SR, however, is a different preparation from lanreotide
Autogel. Lanreotide SR consists of lanreotide incorporated into microparticles (like octreotide LAR) and is injected i.m. In contrast, lanreotide Autogel consists of lanreotide acetate dissolved in water, and is injected deeply s.c. Pharmacokinetic studies of lanreotide Autogel in healthy subjects have shown a release pattern with an almost log-linear decrease of lanreotide serum levels after a single s.c. injection, with a terminal half-life of approximately 4 weeks (Ipsen-Beaufour, unpublished data). There is no evidence of accumulation of lanreotide after multiple doses at any dose either in healthy subjects or in patients. Steady-state serum lanreotide levels are reached after four doses in most patients (IpsenBeaufour, unpublished data). To date, therapeutic lanreotide levels $(>1000 \mathrm{ng} / \mathrm{l})$ in patients under chronic treatment were documented only 30 days after an injection. On the contrary, octreotide levels following a single octreotide LAR injection show a totally different release pattern: immediately after injection there is a small peak followed by an increase in octreotide levels after 7 days reaching a maximum at 28 days, while therapeutic octreotide levels $(>600 \mathrm{ng} / \mathrm{l})$ are maintained up to 42 days (19). These differences in pharmacokinetics between the two depot somatostatin analogues apparently do not result in different efficacy to suppress GH secretion.

In order to obtain the best possible assessment of the GH suppressive effect of both analogues we used detailed $24 \mathrm{~h}$ data analysed with deconvolution analysis. Both depot preparations are registered for clinical use with an injection interval of 4 weeks, implicating that a safe suppression of GH and IGF-I is guaranteed up to 28 days after an injection in octreotide- or lanreotide-sensitive patients. Hence, we considered this time point to be the optimal time point for evaluation of GH secretory profiles in patients on chronic octreotide LAR or lanreotide Autogel treatment. The $24 \mathrm{~h} \mathrm{GH}$ deconvolution analyses illustrate that both longacting somatostatin analogues, octreotide LAR and lanreotide Autogel, induce comparable suppression of GH secretion. Our in vivo data are thus in accord with the in vitro data showing equal binding affinity for the SSTR 2 and 5 (8).

Recently we showed that patients who had well-controlled GH and IGF-I levels with monthly octreotide LAR injections, remained well-controlled in the longterm when the injection interval was extended to 6 weeks (20). Octreotide LAR and lanreotide Autogel showed similar GH and IGF-I suppression at 2, 4 and 6 weeks. Although the measurements after 4 weeks showed a small increase in mean GH and IGF-I concentrations in lanreotide-treated patients, this was not statistically significant. Furthermore, mean simulated GH profiles calculated from eight comparable time points during the $24 \mathrm{~h}$ sampling studies and the IGF-I concentrations were statistically similar to the data obtained at 4 weeks of the withdrawal experiment. Therefore, it might be worthwhile to explore the extension of the 
Pat 1

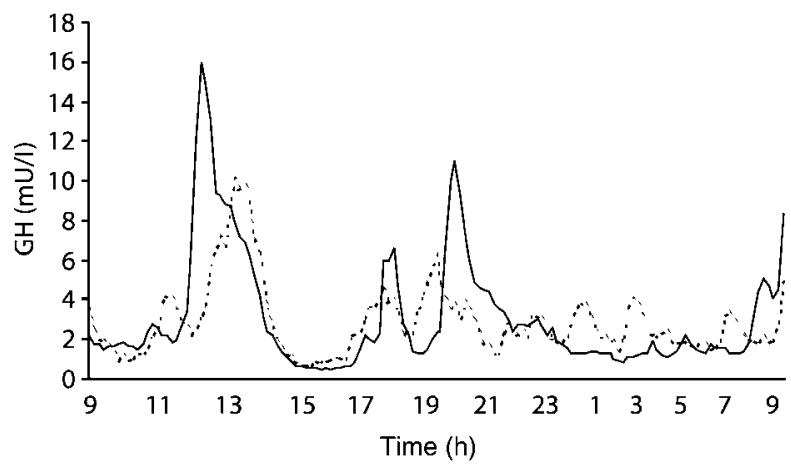

Pat 3

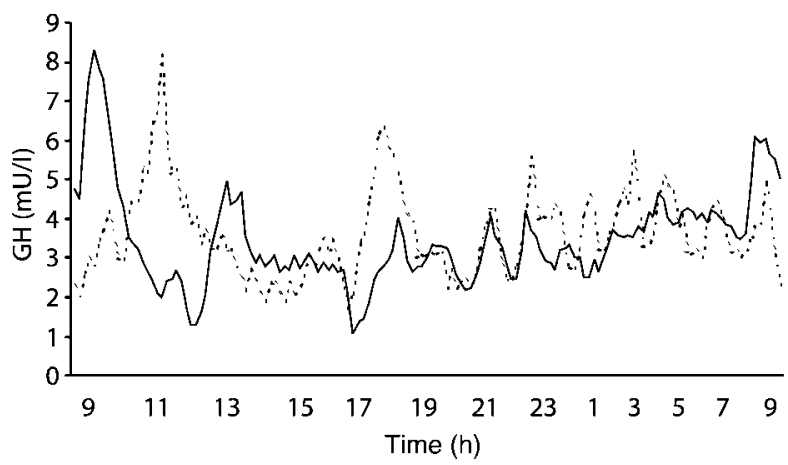

Pat 5

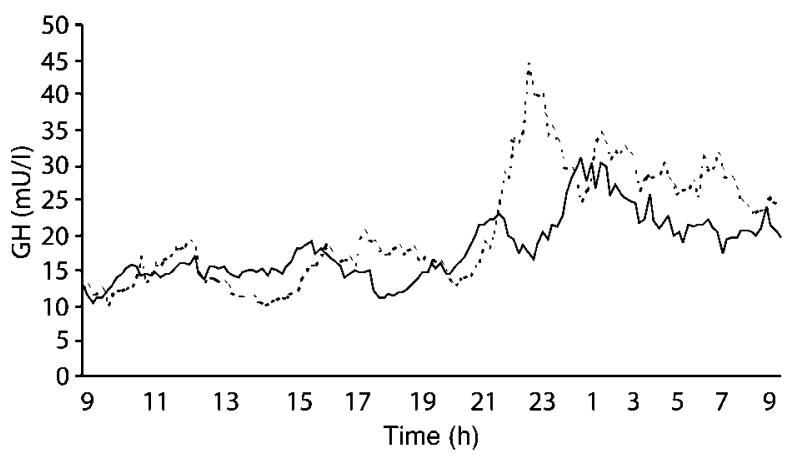

Pat 7

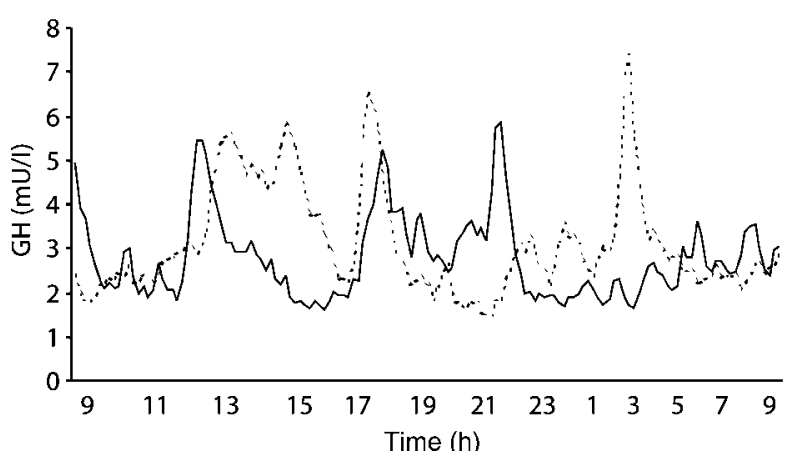

Pat 2

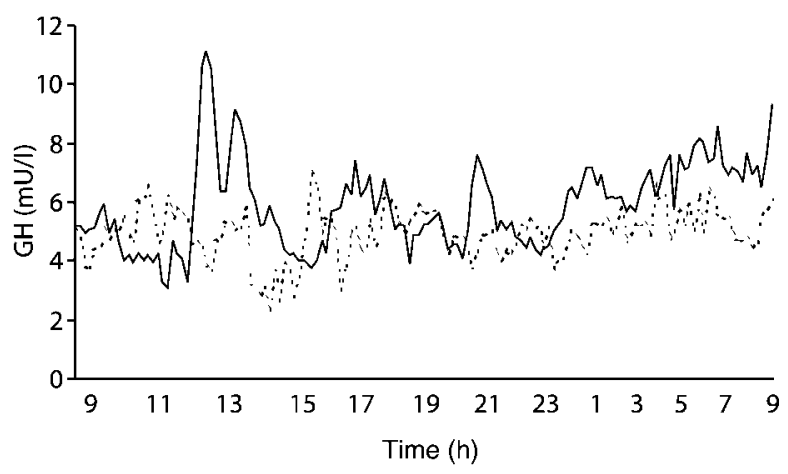

Pat 4

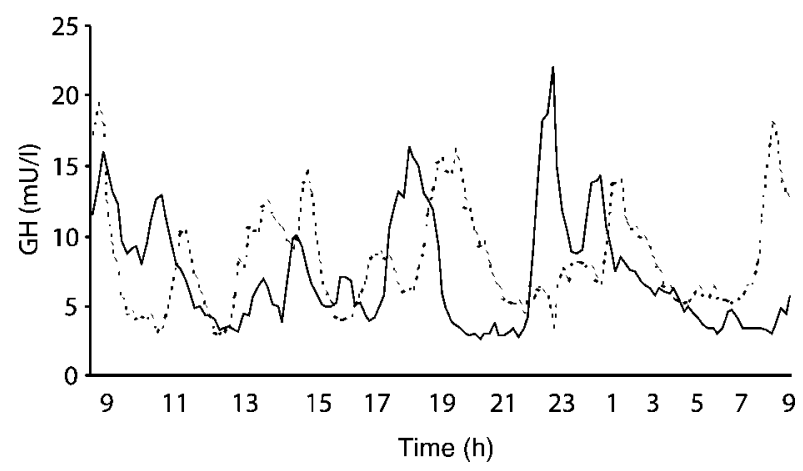

Pat 6

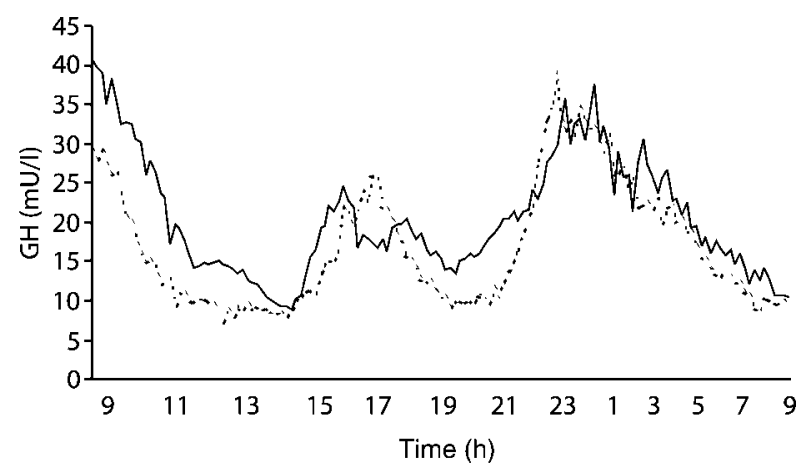

Figure 2 Individual $24 \mathrm{~h}$ GH concentration of each patient (Pat) measured 28 days after the last injection with lanreotide Autogel (solid line) or octreotide LAR (dotted line). 
Table 2 Deconvolution of $24 \mathrm{~h}$ secretory GH profiles in acromegalic patients on treatment with octreotide LAR and lanreotide Autogel. Data are presented as means \pm S.E.M. Statistical comparisons were made with the paired Student's $t$-test.

\begin{tabular}{|c|c|c|c|}
\hline & Octreotide LAR & Lanreotide Autogel & $\boldsymbol{P}$ \\
\hline Secretory half duration (min) & $27.8 \pm 2.9$ & $28.1 \pm 3.3$ & 0.95 \\
\hline Half-life (min) & $16.5 \pm 1.5$ & $15.1 \pm 1.1$ & 0.43 \\
\hline Number of secretory bursts/24h & $36 \pm 1.2$ & $37 \pm 1.7$ & 0.50 \\
\hline Inter-burst interval duration (min) & $40.3 \pm 1.2$ & $38.7 \pm 1.9$ & 0.60 \\
\hline Secretory-burst amplitude (mU/I/min) & $0.27 \pm 0.06$ & $0.29 \pm 0.06$ & 0.51 \\
\hline Burst mass $(\mathrm{mU} / \mathrm{l})$ & $8.59 \pm 2.46$ & $9.02 \pm 2.43$ & 0.80 \\
\hline Basal secretion (mU///24h) & $246 \pm 91$ & $269 \pm 114$ & 0.49 \\
\hline Pulsatile secretion (mU///24 h) & $296 \pm 78$ & $337 \pm 91$ & 0.34 \\
\hline Total secretion $(\mathrm{mU} / \mathrm{l} / 24 \mathrm{~h})$ & $543 \pm 166$ & $606 \pm 199$ & 0.24 \\
\hline
\end{tabular}

Table 3 Mean GH and IGF-I concentrations in acromegalic patients obtained 2, 4 and 6 weeks after injection of octreotide LAR or lanreotide Autogel. Data are shown as mean \pm S.E.M. Statistical calculations were performed with multivariate repeat-measures analysis.

\begin{tabular}{|c|c|c|c|c|}
\hline \multirow[b]{2}{*}{ Weeks after injection } & \multicolumn{2}{|c|}{ Octreotide LAR } & \multicolumn{2}{|c|}{ Lanreotide Autogel } \\
\hline & Mean GH (mU/l) & IGF-I (nmol/I) & Mean GH (mU/l) & IGF-I (nmol/l) \\
\hline 2 & $6.5 \pm 1.8$ & $46 \pm 9$ & $7.1 \pm 1.8$ & $47 \pm 7$ \\
\hline 4 & $7.7 \pm 2.0$ & $40 \pm 4$ & $13.7 \pm 6.9$ & $55 \pm 8$ \\
\hline 6 & $10.1 \pm 2.3$ & $47 \pm 10$ & $10.9 \pm 3.1$ & $53 \pm 8$ \\
\hline
\end{tabular}

For mean $\mathrm{GH}$ levels no differences were found between lanreotide and octreotide treatment $(P=0.53)$ and GH concentrations did not change with time $(P=0.33)$. The interaction term (time $\times$ drug) was non-significant $(P=0.53)$. For IGF-I comparable results were shown. The $P$ values were $0.39,0.43$ and 0.34 respectively.

injection interval for chronic treatment with lanreotide Autogel.

With regard to this observation, one should be cautious to interpret immediate surgical results in patients pre-treated with lanreotide Autogel. Similar to preoperative octreotide LAR treatment, we suggest postponing the postoperative biochemical evaluation to 3 months after the last injection of lanreotide Autogel (19).

Three patients well-controlled on octreotide LAR $20 \mathrm{mg}$ were individually titrated to the maximum dose of lanreotide Autogel. One patient using octreotide LAR $20 \mathrm{mg}$ was subsequently well-controlled with the lowest dose of lanreotide Autogel, e.g. $60 \mathrm{mg} / 4$ weeks. Thus on an individual basis, different pharmacokinetics, for instance bioavailability, could lead to discrepancies in effective dosing.

In conclusion, lanreotide Autogel and octreotide LAR were equally effective in controlling $\mathrm{GH}$ secretion in active acromegaly as measured by suppression of total $24 \mathrm{~h}$ GH secretion and by mean GH levels and IGF-I concentrations. Further analysis should be focused on efficacy in the long-term.

\section{References}

1 Swearingen B, Barker FG II, Katznelson L, Biller BMK, Grinspoon S, Klibanski A et al. Long-term mortality after transsphenoidal surgery and adjunctive therapy for acromegaly. Journal of Clinical Endocrinology and Metabolism 199883 3419-3426.

2 Bates AS, Van't Hoff W, Jones JM \& Clayton RN. An audit of outcome of treatment in acromegaly. Quarterly Journal of Medicine $199386293-299$.
3 Biermasz NR, van Dulken H \& Roelfsema F. Ten-year follow-up results of transsphenoidal microsurgery in acromegaly. Journal of Clinical Endocrinology and Metabolism 200085 4596-4602.

4 Beauregard C, Truong U, Hardy J \& Serri O. Long-term outcome and mortality after transsphenoidal adenomectomy for acromegaly. Clinical Endocrinology 200358 86-91.

5 Melmed S, Casanueva FF, Cavagnini F, Chanson P, Frohman L, Grossman A et al. Guidelines for acromegaly management. Journal of Clinical Endocrinology and Metabolism 200287 4054-4058.

6 Miller GM, Alexander JM, Bikkal HA, Katznelson L, Zervas NT \& Klibanski A. Somatostatin receptor subtype gene expression in pituitary adenomas. Journal of Clinical Endocrinology and Metabolism 199580 1386-1392.

7 Panetta R. \& Patel YC. Expression of mRNA for all five human somatostatin receptors (hSSTR1-5) in pituitary tumors. Life Sciences $199556333-342$.

8 Hofland LJ \& Lamberts SWJ. The pathophysiological consequences of somatostatin receptor internalization and resistance. Endocrine Reviews 200324 28-47.

9 Freda PU. Somatostatin analogs in acromegaly. Journal of Clinical Endocrinology and Metabolism 200287 3013-3018.

10 Chanson P, Boerlin V, Ajzenberg C, Bachelot Y, Benito P, Bringer J et al. Comparison of octreotide acetate LAR and lanreotide SR in patients with acromegaly. Clinical Endocrinology $2000 \mathbf{5 3}$ $577-586$

11 Kendall-Taylor P, Miller M, Gebbie J, Turner S \& al Maskari M. Long-acting octreotide LAR compared with lanreotide SR in the treatment of acromegaly. Pituitary 20003 61-65.

12 Cozzi R, Dallabonzana D, Attanasio R, Barausse M \& Oppizzi G. A comparison between octreotide-LAR and lanreotide-SR in the chronic treatment of acromegaly. European Journal of Endocrinology $1999141267-271$.

13 Turner HE, Vadivale A, Keenan J \& Wass JA. A comparison of lanreotide and octreotide LAR for treatment of acromegaly. Clinical Endocrinology 199951 275-280.

14 Caron P, Beckers A, Cullen DR, Goth MI, Gutt B, Laurberg P et al. Efficacy of the new long-acting formulation of lanreotide (lanreotide Autogel) in the management of acromegaly. Journal of Clinical Endocrinology and Metabolism 2002 87 99-104. 
15 Biermasz NR, Pereira AM, Frolich M, Romijn JA, Veldhuis JD \& Roelfsema F. Octreotide represses secretory-burst mass and nonpulsatile secretion but does not restore event frequency or orderly GH secretion in acromegaly. American Journal of Physiology. Endocrinology and Metabolism 2004286 E25-E30.

16 Veldhuis JD, Moorman J \& Johnson ML. Deconvolution analysis of neuroendocrine data: waveform-independent methods and application. Methods in Neurosciences 199420 279-325.

17 Veldhuis JD \& Johnson ML. Specific methodological approaches to selected contemporary issues in deconvolution analysis of pulsatile neuroendocrine data. Methods in Neurosciences $1995 \mathbf{2 8}$ 28-92.

18 Flogstad AK, Halse J, Bakke S, Lancranjan I, Marbach P, Bruns C et al. Sandostatin LAR in acromegalic patients: long term treatment. Journal of Clinical Endocrinology and Metabolism 199782 23-28.

19 Lancranjan I, Bruns C, Grass P, Jaquet P, Jervell J, Kendall-Taylor P et al. Sandostatin LAR: a promising therapeutic tool in the management of acromegalic patients. Metabolism 199645 67-71.

20 Biermasz NR, van den Oever NC, Frolich M, Pereira Arias AM, Smit JW, Romijn JA et al. Sandostatin LAR in acromegaly: a 6-week injection interval suppresses GH secretion as effectively as a 4-week interval. Clinical Endocrinology 200358 288-295.

Received 22 August 2003

Accepted 23 December 2003 